\title{
Protective Effect of Morus alba Leaf Extract on $N$-Nitrosodiethylamine-induced Hepatocarcinogenesis in Rats
}

\author{
MAŁGORZATA KUJAWSKA ${ }^{1}$, MAŁGORZATA EWERTOWSKA ${ }^{1}$, TERESA ADAMSKA ${ }^{1}$, EWA IGNATOWICZ ${ }^{2}$, \\ EWA FLACZYK ${ }^{3}$, MONIKA PRZEOR $^{3}$, MONIKA KURPIK $^{1}$ and JADWIGA JODYNIS LIEBERT ${ }^{1}$ \\ ${ }^{1}$ Department of Toxicology, Poznan University of Medical Sciences, Poznań, Poland; \\ ${ }^{2}$ Department of Pharmaceutical Biochemistry, Poznan University of Medical Sciences, Poznań, Poland; \\ ${ }^{3}$ Department of Food Service and Catering, Poznań University of Life Sciences, Poznań, Poland
}

\begin{abstract}
Background/Aim: The leaves of white mulberry (Morus alba L.) contain various polyphenolic compounds possessing strong antioxidant activity and anticancer potential. This study was designed to investigate the chemopreventive effect of aqueous extract of mulberry leaves against $N$-nitrosodiethylamine (NDEA)-induced liver carcinogenesis. Materials and Methods: Wistar rats were divided into four groups: control, mulberry extract-treated, NDEA-treated, and mulberry extract plus NDEA-treated. Mulberry extract was given in the diet $(1,000 \mathrm{mg} / \mathrm{kg}$ b.w./day); NDEA was given in drinking water. Results: Mulberry extract reduced the incidence of hepatocellular carcinoma, dysplastic nodules, lipid peroxidation, protein carbonyl formation, and DNA degradation. Treatment with mulberry leaf extract along with NDEA challenge did not affect the activity of antioxidant enzymes and glutathione content. Conclusion: Treatment with mulberry leaf extract partially protected the livers of rats from NDEA-induced hepatocarcinogenesis and a direct antioxidant mechanism appears to contribute to its anticarcinogenic activity.
\end{abstract}

Since primary liver cancer is the third leading cause of cancer mortality worldwide, chemopreventive strategies aimed at reducing its risk or delaying its onset are highly desirable. The most common type of primary liver cancer is an inflammation-associated cancer developing from hepatocytes, hepatocellular carcinoma (HCC). Hepatocarcinogenesis progresses from chronic intrahepatic inflammation within the

Correspondence to: Małgorzata Kujawska, Department of Toxicology, Poznan University of Medical Sciences, 30 Dojazd Str., 60-631, Poznań, Poland. E-mail: kujawska@ump.edu.pl

Key Words: Mulberry, Morus alba L., lipid peroxidation, protein carbonyls, comet assay. state of oxidative stress, which results in continuous cellular injury, necrosis and regeneration along with a genotoxic effect $(1,2)$. Despite a clear viral etiology, HCC is also mediated through exposure to hepatocarcinogens such as nitrosamines, which cause the generation of radicals or cellular mitochondrial dysfunction (2).

White mulberry (Morus alba) leaves contain abundant varieties of polyphenols, including chlorogenic acid, rutin, isoquercitrin, quercetin, astragalin and kaempferol, which are considered strong antioxidants (3). Mulberry leaf extract has been reported to scavenge 1,1-diphenyl-2-picryl-hydrazyl radical and prevent lipid peroxidation in rabbit and human low-density lipoproteins (4). Its antioxidant effect has been also revealed in streptozotocin-induced diabetic rats (5). Anticancer properties of mulberry leaf polyphenols have been demonstrated in numerous assays with various types of human cancer cells of colon (6), liver (7), breast (6), and lung (8), and the underlying mechanisms including antioxidant, antiinflammatory, and proliferative, and cytotoxic activity have been shown. However, to the best of our knowledge, the anticancer effect in an animal model has not yet been investigated.

The present study was designed to evaluate the chemopreventive effect of mulberry leaf extract on $\mathrm{N}$ nitrosodiethylamine (NDEA)-induced liver carcinogenesis in rats. This experimental model of hepatocarcinogenesis, due to histological and biochemical similarities between rodents and human hepatic lesions, is widely used in chemoprevention studies (9).

\section{Materials and Methods}

Materials. Dried and crushed M. alba L. (var. wielkolistna zolwinska) leaves were mixed with water $\left(80-90^{\circ} \mathrm{C}\right)$ using a counterflow process $(1: 10 \mathrm{w} / \mathrm{w})$ and subjected to continuous extraction in a twin-screw extractor (IBPRS, Poznan, Poland). The resulting extract was then concentrated using a vacuum periodic spherical evaporator 
(WWA20; Spomasz, Pleszew, Poland) and a spray dryer (SR16, Niro Atomizer, Søborg, Denmark) (10). The content of polyphenolic compounds was determined previously and is given in Table I (10).

Experimental design. Thirty-two male Wistar rats weighing $250 \pm 15 \mathrm{~g}$ (12 weeks old) bred at the Department of Toxicology, Poznan University of Medical Sciences, were randomly assigned to four different treatment groups of eight animals each. Experimental groups were treated for 13 weeks as follows: Group 1: Control rats fed the standard diet; group 2: rats given $M$. alba extract at a concentration of $10 \mathrm{~g} / \mathrm{kg}$ feed; group 3: rats receiving $0.01 \%$ NDEA in drinking water; group 4: rats treated with $10 \mathrm{~g} / \mathrm{kg}$ feed $M$. alba extract along with the administration of $0.01 \%$ NDEA in drinking water. On the basis of the feed consumption and the nominal dietary concentration of $M$. alba extract, the calculated mean daily intake of test substance was about $1,000 \mathrm{mg} / \mathrm{kg} \mathrm{b.w./day.} \mathrm{At} \mathrm{the} \mathrm{end} \mathrm{of} \mathrm{the} 13$-week treatment, the animals were fasted overnight and then were anesthetized by ketamine/xylazine (100/7.5 mg/kg b.w., i.p.). The animal experiment was approved by the Local Animal Ethics Committee (permission number 28/2011).

Sample collection. Livers were rapidly removed and processed for histopathological and biochemical examination. For histopathological analysis, a slice of the liver was fixed in $4 \%$ paraformaldehyde. Another portion of the liver was homogenized in buffered Tris/sucrose solution $(\mathrm{pH}$ 7.55) for differential centrifugation according to the standard procedure to obtain the cytosolic fraction. For glutathione, lipid peroxidation and protein carbonyl determinations, as well as comet assay, other liver tissue sections were homogenized separately. For each fraction, the protein concentration was determined using Folin-Ciocalteu reagent.

Histopathology. After formalin fixation, liver specimens were dehydrated and paraffin impregnated. Paraffin blocks were sectioned at $4 \mu \mathrm{m}$ then stained with hematoxylin and eosin and then cells and tissues were examined using light microscopy.

Biochemical assays. The level of microsomal lipid peroxidation was assayed by measuring thiobarbituric acid reactive substances (TBARS) (11). Protein carbonyl concentration was assessed using a commercial enzyme-linked immunosorbent assay kit from BioCell Corporation, Auckland, New Zealand.

An alkaline comet assay was conducted according to the method of Hartmann et al. (12). After cell lysis, DNA unwinding, electrophoresis and neutralization, the slides were stained with ethidium bromide. Images of comets from a Zeiss fluorescence microscope (magnification $\times 400$ ) were captured with a digital camera and scored into 5 groups according to the degree of DNA damage (13). Reduced glutathione content was assessed by its reaction with Ellman's reagent (14).

Antioxidant enzyme activities were determined in the liver cytosol using spectrophotometric methods. Superoxide dismutase assay was based on its ability to inhibit spontaneous epinephrine oxidation (15). Catalase activity was assessed by the measurement of the rate of $\mathrm{H}_{2} \mathrm{O}_{2}$ decomposition (15). Glutathione peroxidase activity was determined according to Mohandas et al. (16), with hydrogen peroxide as a substrate; the rate of the NADPH disappearance at $340 \mathrm{~nm}$ was a measure of the enzyme activity. Glutathione reductase activity was assayed by measuring NADPH oxidation at $340 \mathrm{~nm}$ in the presence of oxidized glutathione (16).
Table I. Content of polyphenolic compounds in Morus alba leaf aqueous extract (10).

\begin{tabular}{lc}
\hline Compound & Content $(\mathrm{g} / 100 \mathrm{~g})$ \\
\hline Gallic acid & 0.28 \\
Protocatechuic acid & 0.08 \\
$p$-Hydroxybenzoic acid & 0.11 \\
Vanillic acid & 0.42 \\
Chlorogenic acid & 2.33 \\
Caffeic acid & 0.66 \\
$p$-Coumaric & 0.12 \\
Ferulic acid & 0.09 \\
Sinapic acid & 0.11 \\
Total phenolic acids & 4.27 \\
Rutin & 0.90 \\
Quercetin 3- $\beta$-D-glucoside & 0.47 \\
Kaempferol 3- $\beta$-D- glucopyranoside & 4.00 \\
Total flavonols & 1.56 \\
\hline
\end{tabular}

Glutathione-S-transferase activity measurement was based on the determination of 1-chloro-2,4-dinitrobenzene conjugate formed in a glutathione (GSH)-coupled reaction (16). Paraoxonase-1 activity was measured with phenylacetate as a substrate; the rate of phenol generation was a measure of the enzyme activity (17). The data are expressed as the mean $\pm \mathrm{SD}$. One-way analysis of variance (ANOVA) followed by the Tukey-Kramer multiple comparisons test was used. A value of $p<0.05$ was considered statistically significant.

\section{Results}

Microscopic evaluation. Histopathological examination of liver sections from controls and rats treated with the mulberry extract revealed normal architecture (Figure 1A and B). Liver tissue of NDEA-administered animals showed the presence of hepatocellular carcinoma, dysplastic nodules and cirrhosis (Figure 1C). Administration of the M. alba extract resulted in a modest improvement of histological architecture of the liver tissue (Figure 1D) and reduced the incidence of hepatocellular carcinoma and dysplastic nodules (Table II).

Oxidative stress evaluation. The effects of $M$. alba leaf extract on the levels of oxidative modification of lipids, proteins and DNA are illustrated by the data in Table III. In rats exposed to NDEA, a significant increase in protein oxidation, of more than six-fold, as well as in lipid peroxidation and DNA degradation by $142 \%$ and $64 \%$, respectively, was observed. Mulberry extract administration to NDEA-treated rats caused a significant decline in levels of protein carbonyls and TBARS, as well as a decrease in comet size by $75 \%, 66 \%$, and $6 \%$, respectively, as compared to those in rats treated with NDEA alone. In rats treated with NDEA alone, the hepatic GSH level was increased by $28 \%$ as compared to that in the controls (Table III). Administration 

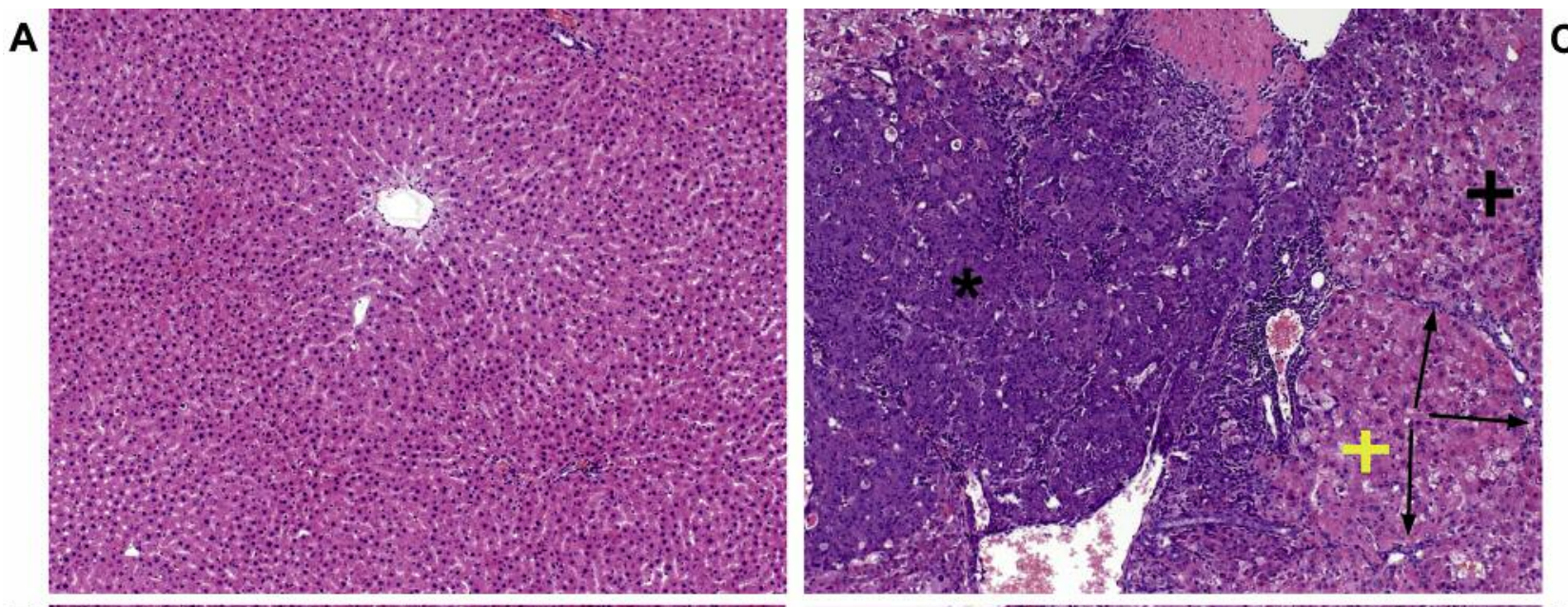

B
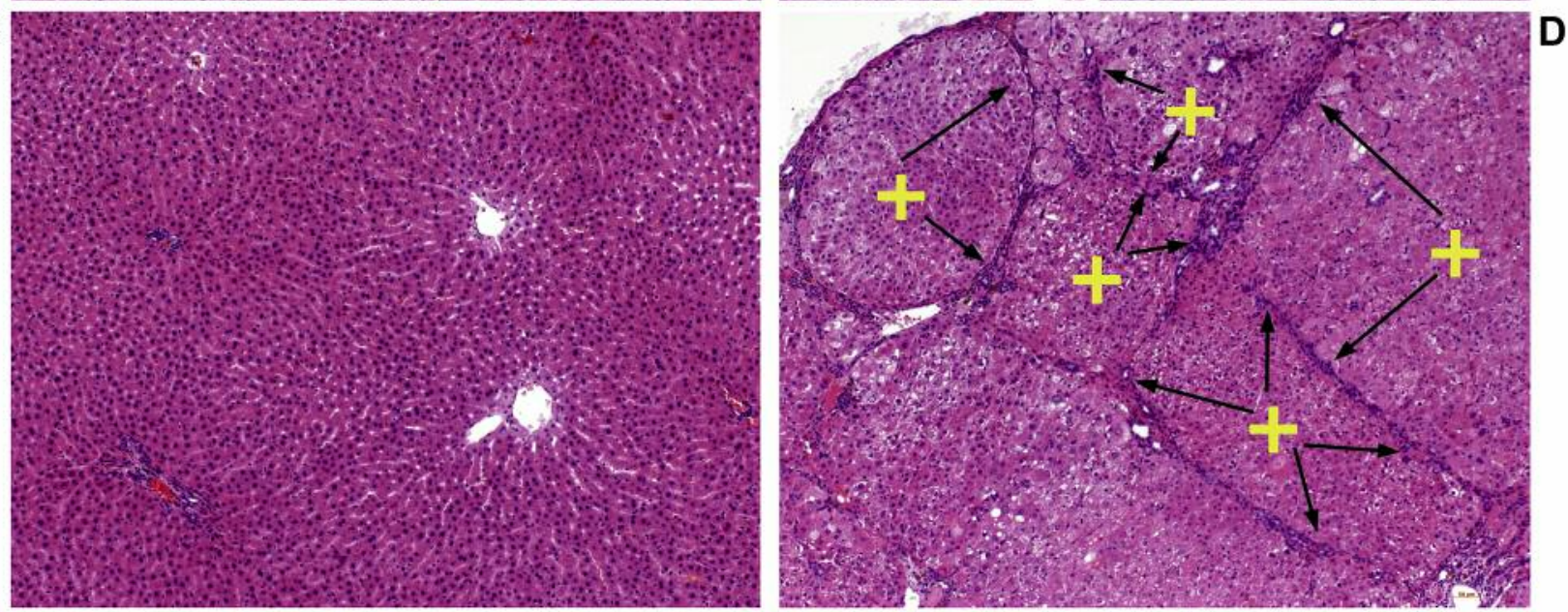

Figure 1. Representative images (magnification $\times 100$ ) of hematoxylin and eosin staining in liver sections from: control rats, showing normal liver architecture (A); rats treated with mulberry extract alone, also showing normal liver architecture (B); rats treated with (NDEA) $N$ nitrosodiethylamine alone, showing hepatocellular carcinoma tumor (asterisk) with surrounding liver tissue (black cross), and cirrhosis with deposition of connective tissue (arrows) surrounding a nodule (yellow cross) (C); and rats treated with mulberry extract plus NDEA, showing cirrhosis with deposition of connective tissue (arrows) surrounding nodules (yellow crosses) (D).

of mulberry leaf extract to NDEA-challenged rats slightly reduced the level of GSH; however, the difference was not statistically significant. The response of hepatic antioxidant enzymes to NDEA administration was diverse (Table IV). The superoxide dismutase and catalase activities remained unchanged. The activities of glutathione-S-transferase and glutathione reductase were increased by $49 \%$ and $82 \%$, respectively, whereas the activity of glutathione peroxidase was inhibited by $68 \%$, compared with those in the control rats. Nevertheless, supplementation with $M$. alba leaf extract did not have a significant effect on NDEA-induced changes in activity of these enzymes. NDEA also caused an increase in the hepatic paraoxonase- 1 activity, by $38 \%$, which remained unchanged after co-treatment with mulberry extract.

\section{Discussion}

Several reports have demonstrated that oxidative stress may be linked to carcinogenesis in the case of NDEA-induced HCC $(9,18)$. Previously, we demonstrated antioxidant activity of $M$. alba leaf extract in assays based on the scavenging of stable 2,2-diphenyl-1-picryl hydrazyl (DPPH) and 2,2'azinobis-3-ethylbenzo thiazoline-6-sulfonate (ABTS) radicals, as well as the ability to chelate iron (II) (10). In this study, we investigated whether the extract tested can provide some protection against NDEA-induced hepatocarcinogenesis in rats. Microscopic studies of livers stained with hematoxylin and eosin revealed extensive tissue damage as evidenced by cirrhosis, dilatation of bile ducts and ballooning degeneration, 
in vivo $30: 807-812(2016)$

Table II. Effect of mulberry extract on histopathological parameters in liver of rats treated with/without N-nitrosodiethylamine (NDEA).

\begin{tabular}{lccccc}
\hline Parameter & \multicolumn{4}{c}{ Treatment, $\mathrm{n} / \mathrm{N}$} \\
\cline { 2 - 5 } & $\mathrm{N}$ & Control & Mulberry extract & NDEA & Mulberry extract + NDEA \\
\hline Hepatocellular carcinoma & 8 & $0 / 8$ & $0 / 8$ & $2 / 8$ & $1 / 8$ \\
Dysplastic nodules & 8 & $0 / 8$ & $0 / 8$ & $2 / 8$ & $0 / 8$ \\
Cirrhosis & 8 & $0 / 8$ & $0 / 8$ & $3 / 8$ & $3 / 8$ \\
Nodulation of the liver & 8 & $0 / 8$ & $0 / 8$ & $5 / 8$ & $5 / 8$ \\
Bile duct dilatation & 8 & $0 / 8$ & $0 / 8$ & $1 / 8$ & $5 / 8$ \\
Cholestasis & 8 & $0 / 8$ & $0 / 8$ & $3 / 8$ & $4 / 8$ \\
Ballooning degeneration & 8 & $0 / 8$ & & $4 / 8$ \\
\hline
\end{tabular}

Table III. Effect of mulberry extract on lipid peroxidation (LPO), protein carbonyls (PCs) concentration, DNA damage and reduced glutathione $(G S H)$ level in liver of rats treated with/without N-nitrosodiethylamine (NDEA).

\begin{tabular}{lcccc}
\hline Treatment & LPO (nmol TBARS/g protein) & PC (nmol/mg protein) & DNA damage (arbitrary points) & GSH $(\mu \mathrm{mol} / \mathrm{g}$ tissue $)$ \\
\hline Control & $19.16 \pm 2.96$ & $0.12 \pm 0.03$ & $77.98 \pm 8.37$ & $2.82 \pm 0.24$ \\
Mulberry extract & $20.17 \pm 2.45$ & $0.13 \pm 0.03$ & $81.02 \pm 6.96$ & $2.73 \pm 0.27$ \\
NDEA & $46.37 \pm 5.72(\uparrow 142 \%)^{\mathrm{a}}$ & $0.87 \pm 0.23(\uparrow 646 \%)^{\mathrm{a}}$ & $128.11 \pm 4.04(\uparrow 64 \%)^{\mathrm{a}}$ & $3.62 \pm 0.72(\uparrow 28 \%)^{\mathrm{a}}$ \\
Mulberry extract +NDEA & $15.96 \pm 1.77(\downarrow 66 \%)^{\mathrm{b}}$ & $0.22 \pm 0.06(\downarrow 75 \%)^{\mathrm{b}}$ & $120.15 \pm 9.58(\uparrow 54 \%)^{\mathrm{a}}(\downarrow 6 \%)^{\mathrm{b}}$ & $3.30 \pm 0.21$ \\
\hline
\end{tabular}

TBARS: Thiobarbituric acid-reactive substances. Data are means \pm SD $(\%$ change $) . \mathrm{n}=8$. Significantly different from ${ }^{\mathrm{a}}$ control, bNDEA-treated group at $p<0.05$.

Table IV. Effect of mulberry extract on hepatic antioxidant enzyme activity in rats treated with/without N-nitrosodiethylamine (NDEA).

\begin{tabular}{lcccc}
\hline & & \multicolumn{3}{c}{ Treatment } \\
\cline { 3 - 5 } Parameter & Control & Mulberry extract & NDEA & Mulberry extract + NDEA \\
\hline SOD (U/mg protein) & $64.99 \pm 16.33$ & $69.78 \pm 8.19$ & $62.98 \pm 14.15$ & $69.54 \pm 18.62$ \\
CAT (U/mg protein) & $7.97 \pm 1.13$ & $6.79 \pm 1.11$ & $8.08 \pm 1.81$ & $9.19 \pm 1.38$ \\
GPx (nmol NADPH/min/mg protein) & $895.76 \pm 128.10$ & $922.78 \pm 184.53$ & $283.08 \pm 104.18(\downarrow 68 \%)^{\mathrm{a}}$ & $309.00 \pm 104.41(\downarrow 66 \%)^{\mathrm{a}}$ \\
GR (nmol NADPH/min/mg protein) & $59.78 \pm 18.12$ & $71.97 \pm 17.81$ & $108.88 \pm 30.81(\uparrow 82 \%)^{\mathrm{a}}$ & $114.62 \pm 37.73(\uparrow 92 \%)^{\mathrm{a}}$ \\
GST $(\mathrm{nmol} \mathrm{CDNB} / \mathrm{min} / \mathrm{mg}$ protein) & $1332.77 \pm 145.82$ & $1241.53 \pm 194.22$ & $1981.09 \pm 209.86(\uparrow 49 \%)^{\mathrm{a}}$ & $1980.96 \pm 261.68(\uparrow 49 \%)^{\mathrm{a}}$ \\
PON1 $(\mu \mathrm{mol} \mathrm{phenol} / \mathrm{min} / \mathrm{mg}$ protein) & $2.67 \pm 0.72$ & $2.31 \pm 0.71$ & $3.68 \pm 1.06(\uparrow 38 \%)^{\mathrm{a}}$ & $3.50 \pm 0.90$ \\
\hline
\end{tabular}

SOD: Superoxide dismutase; CAT: catalase; GPx: glutathione peroxidase; NADPH: nicotinamide adenine dinucleotide phosphate; GR: glutathione reducatse; GST: glutathione-S-transferase; CDNB: 1-chloro-2,4-dinitrobenzene; PON1: paraoxonase-1. Data are means \pm SD (\% change). $\mathrm{n}=8$. aSignificantly different from control at $p<0.05$. Results are mean \pm SD. $\mathrm{n}=8$.

as well as the presence of HCC and dysplastic nodules in rats treated with NDEA. These histological findings corroborate those from other studies $(19,20)$. Shen et al. reported that in rats exposed to NDEA, small pre-neoplastic focal lesions undergo malignant transformation, with the formation of neoplastic nodules and ultimately HCC (21). The mulberry extract inhibited the malignant transformation process since the incidence of HCC and dysplastic nodules was reduced in the livers of rats receiving the extract and NDEA. Additionally, the extract partially improved the hepatic cellular architecture. It has been reported by others that polyphenols such as: morin (22), apigenin (19), green tea polyphenols (21), as well as a polyphenolics-rich extract from Acacia nilotica bark (23), moderately improved the hepatocellular structure 
damaged by NDEA treatment. It is widely accepted that NDEA undergoes metabolic activation by cytochrome $P 450$ 2E1 (CYP2E1) and consequently oxygen radical by-products and the active ethyl radical metabolite $\left(\mathrm{CH}_{3} \mathrm{CH}_{2}{ }^{+}\right)$are formed. NDEA-generated reactive oxygen species cause oxidative modifications of macromolecules which, together with DNA alkylation can initiate hepatocarcinogenesis (20). Since hepatic oxidative stress in rats following NDEA administration can promote hepatocarcinogenesis, the suppressive effects of mulberry leaf extract on the development of HCC may be due to its ability to scavenge free radicals or to the induction of hepatic antioxidant defense.

The current study confirmed that oxidative stress contributed to NDEA-induced hepatocarcinogenesis, as was evidenced by the increase in the content of protein carbonyls and lipid peroxidation products and damage to DNA. Aparicio-Bautista et al. demonstrated that NDEA-induced thiol protein oxidation was involved in the early stages of hepatocarcinogenesis (24). The increase of the hepatic level of protein carbonyls in NDEA-treated animals was also corroborated by Bishayee et al. (25). NDEA-induced products of oxidative damage to lipids can attack DNA, among other cellular targets, thereby promoting mutagenicity and carcinogenicity (20). The electrophilic by-product generated during NDEA biotransformation has been reported to alkylate DNA to form pro-mutagenic oxidized bases and to initiate DNA fragmentation (26). In the current experiment, NDEA increased DNA damage, as was evidenced by the increase in comet tail length. The administration of mulberry leaf extract markedly inhibited protein and lipid oxidation and ameliorated DNA damage, which can be interpreted as a suppression of NDEA-induced oxidative stress. These findings fully corroborate our earlier in vitro study in which we found a high capacity of the extract to reduce ABTS cation and DPPH: $41.36 \mu \mathrm{Mol}$ Trolox $\mathrm{g}^{-1}$ dry weight and $137.60 \mu \mathrm{Mol}$ Trolox $\mathrm{g}^{-1}$ dry weight, respectively (10). Similar results regarding protective effects of mulberry leaf extracts against oxidative damage of macromolecules have been reported in the liver of high fat diet-induced obese mice (27).

Glutathione serves numerous important functions including neutralization of free radicals, detoxification of toxic electrophiles and peroxides (or other oxidizing reagents), as well as mediation in S-glutathionylation (28). Marinho et al. reported that chronic exposure to NDEA caused the induction of gamma-glutamyl transferase and gamma-glutamylcysteine synthetase activity, resulting in an increase in the content of GSH in the liver (29). In the present study, the enhancement of hepatic GSH level in NDEA-treated rats was accompanied by an increase in activities of glutathione S-transferase and glutathione reductase, and these findings are consistent with those of Santos et al. (18). Furthermore, we noticed an increase in the activity of paraoxonase 1 , an enzyme involved in protection against lipid peroxidation (21). Overall, these results can be interpreted as a cytoprotective response against electrophiles and oxidants (30). Treatment with the mulberry leaf extract along to NDEA challenge did not affect the activity of antioxidant enzymes or glutathione content.

Taken together, the histological and biochemical findings of the present study demonstrate that treatment with mulberry extract partially suppressed NDEA-initiated hepatocarcinogenesis and the appearance of pre-neoplastic lesions. Since the extract tested has been reported to possess strong free radical-scavenging properties in vitro and we showed its ability to prevent oxidative damage of macromolecules in rats, it could be suggested that a direct antioxidant mechanism contributes to its anticarcinogenic activity. However, further research should be undertaken to examine whether the anti-inflammatory mechanism is also responsible for the chemopreventive effect. In conclusion, M. alba leaf extract appears to be an attractive candidate for chemoprevention of liver cancer.

\section{References}

1 Singh S, Singh PP, Roberts LR and Sanchez W: Chemopreventive strategies in hepatocellular carcinoma. Nat Rev Gastroenterol Hepatol 11: 45-54, 2014.

2 Mastron JK, Siveen KS, Sethi G and Bishayee A: Silymarin and hepatocellular carcinoma: a systematic, comprehensive, and critical review. Anticancer Drugs 26: 475-486, 2015.

3 Lee WJ and Choi SW: Quantitative changes of polyphenolic compounds in mulberry (Morus alba L.) Leaves in relation to varieties, harvest period, and heat processing. Prev Nutr Food Sci 17: 280-285, 2012.

4 Chao PY, Lin KH, Chiu CC, Yang YY, Huang MY and Yang $\mathrm{CM}$ : Inhibitive effects of mulberry leaf-related extracts on cell adhesion and inflammatory response in human aortic endothelial cells. Evid Based Complement Alternat Med 2013: 267217, 2013.

5 Andallu B, Kumar AV and Varadacharyulu NC: Oxidative stress in streptozocin-diabetic rats: Amelioration by mulberry (Morus indica L.) leaves. Chin J Integr Med doi:10.1007/s11655-0121234-4, 2012.

6 Deepa M, Sureshkumar T, Satheeshkumar PK and Priya S: Antioxidant rich Morus alba leaf extract induces apoptosis in human colon and breast cancer cells by the downregulation of nitric oxide produced by inducible nitric oxide synthase. Nutr Cancer 65: 305-310, 2013.

7 Naowaratwattana W, De-Eknamkul W and De Mejia EG: Phenolic-containing organic extracts of mulberry (Morus alba L.) leaves inhibit HepG2 hepatoma cells through $\mathrm{G}_{2} / \mathrm{M}$ phase arrest, induction of apoptosis, and inhibition of topoisomerase II $\alpha$ activity. J Med Food 13: 1045-1056, 2010.

8 Chen PN, Chu SC, Chiou HL, Kuo WH, Chiang CL and Hsieh YS: Mulberry anthocyanins, cyanidin 3-rutinoside and cyanidin 3-glucoside, exhibited an inhibitory effect on the migration and invasion of a human lung cancer cell line. Cancer Lett 235: 248$259,2006$. 
9 Paula Santos N, Colaço A, Gil da Costa RM, Manuel Oliveira M, Peixoto Fand Alexandra Oliveira P: N-Diethylnitrosamine mouse hepatotoxicity: time-related effects on histology and oxidative stress. Exp Toxicol Pathol 66: 429-436, 2014.

10 Flaczyk E, Kobus-Cisowska J, Przeor M, Korczak J, Remiszewski M, Korbas E and Buchowski M: Chemical characterization and antioxidative properties of Polish variety of Morus alba L. leaf aqueous extracts from the laboratory and pilot-scale processes. Agricultural Sciences 4: 141-147, 2013.

11 Sanz MJ, Ferrandiz ML, Cejudo M, Terencio MC, Gil B, Bustos G, Ubeda A, Gunasegaran R and Alcaraz MJ: Influence of a series of natural flavonoids on free radical generating systems and oxidative stress. Xenobiotica 24: 689-699, 1994.

12 Hartmann A, Agurell E, Beevers C, Brendler-Schwaab S Burlinson B, Clay P, Collins A, Smith A, Speit G, Thybaud V and Tice RR: Recommendations for conducting the in vivo alkaline comet assay. Mutagenesis 18: 45-51, 2003.

13 Collins AR: The comet assay for DNA damage and repair: principles, applications and limitations. Mol Biotechnol 26: 249261,2004

14 Sedlak J and Lindsay RH: Estimation of total, protein-bound, and nonprotein sulfhydryl groups in tissue with Ellman's reagent. Anal Biochem 25: 192-205, 1968.

15 Jodynis-Liebert $\mathrm{J}$, Murias $\mathrm{M}$ and Błoszyk E: Effect of sesquiterpene lactones on antioxidant enzymes and some drugmetabolizing enzymes in rat liver and kidney. Planta Med 66: 199-205, 2000.

16 Mohandas J, Marshall JJ, Duggin GG, Horvath JS and Tiller DJ: Low activities of glutathione-related enzymes as factors in the genesis of urinary bladder cancer. Cancer Res 44: 5086-5091, 1984.

17 Jurek A, Turyna B, Kubit P and Klein A: LDL susceptibility to oxidation and HDL antioxidant capacity in patients with renal failure. Clin Biochem 39: 19-27, 2006.

18 Santos NP, Pereira IC, Pires MJ, Lopes C, Andrade R, Oliveira MM, Colaço A, Peixoto F and Oliveira PA: Histology, bioenergetics and oxidative stress in mouse liver exposed to $\mathrm{N}$ diethylnitrosamine. In Vivo 26: 921-929, 2012.

19 Ali F, Rahul, Naz F, Jyoti S and Siddique YH: Protective effect of apigenin against $N$-nitrosodiethylamine (NDEA)-induced hepatotoxicity in albino rats. Mutat Res Genet Toxicol Environ Mutagen. 767: 13-20, 2014

20 Arul D and Subramanian P: Inhibitory effect of naringenin (citrus flavonone) on $N$-nitrosodiethylamine induced hepatocarcinogenesis in rats. Biochem Biophys Res Commun 434: 203-209, 2013.
21 Shen T, Khor SC, Zhou F, Duan T, Xu YY, Zheng YF, Hsu S, DE Stefano J, Yang J, Xu LH and Zhu XQ: Chemoprevention by lipid-soluble tea polyphenols in diethylnitrosamine/ phenobarbital-induced hepatic pre-cancerous lesions. Anticancer Res 34: 683-693, 2014.

22 MadanKumar P, NaveenKumar P, Manikandan S, Devaraj H and Niranjali Devaraj S: Morin ameliorates chemically induced liver fibrosis in vivo and inhibits stellate cell proliferation in vitro by suppressing Wnt/ $\beta$-catenin signaling. Toxicol Appl Pharmacol 277: 210-220, 2014

23 Singh BN, Singh BR, Sarma BK and Singh HB: Potential chemoprevention of $\mathrm{N}$-nitrosodiethylamine-induced hepatocarcinogenesis by polyphenolics from Acacia nilotica bark. Chem Biol Interact 181: 20-28, 2009.

24 Aparicio-Bautista DI, Pérez-Carreón JI, Gutiérrez-Nájera N, Reyes-Grajeda JP, Arellanes-Robledo J, Vásquez-Garzón VR, Jiménez-García MN and Villa-Treviño S: Comparative proteomic analysis of thiol proteins in the liver after oxidative stress induced by diethylnitrosamine. Biochim Biophys Acta 1834: 2528-2538, 2013

25 Bishayee A, Barnes KF, Bhatia D, Darvesh AS and Carroll RT: Resveratrol suppresses oxidative stress and inflammatory response in diethylnitrosamine-initiated rat hepatocarcinogenesis. Cancer Prev Res 3: 753-763, 2010.

26 Verna L, Whysner J and Williams GM: $N$-Nitrosodiethylamine mechanistic data and risk assessment: bioactivation, DNAadduct formation, mutagenicity, and tumor initiation. Pharmacol Ther 71: 57-81, 1996.

27 Lim HH, Yang SJ, Kim Y, Lee M and Lim Y: Combined treatment of mulberry leaf and fruit extract ameliorates obesityrelated inflammation and oxidative stress in high fat diet-induced obese mice. J Med Food 16: 673-680, 2013.

28 Paulsen CE and Carroll KS: Cysteine-mediated redox signaling: chemistry, biology, and tools for discovery. Chem Rev 113: 4633-4679, 2013.

29 Marinho HS, Baptista M and Pinto RE: Glutathione metabolism in hepatomous liver of rats treated with diethylnitrosamine. Biochim Biophys Acta 1360: 157-168, 1997.

30 Satoh T, McKercher SR and Lipton SA: Nrf2/ARE-mediated antioxidant actions of pro-electrophilic drugs. Free Radic Biol Med 65: 647-657, 2013

Received July 21, 2016

Revised August 17, 2016 Accepted August 18, 2016 